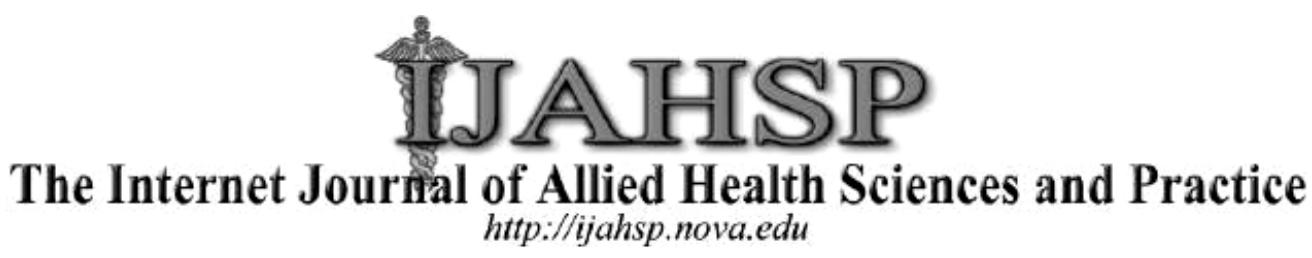

A Peer Reviewed Publication of the College of Allied Health \& Nursing at Nova Southeastern University Dedicated to allied health professional practice and education

http://ijahsp.nova.edu Vol. 8 No. 2 ISSN 1540-580X

\title{
Neurodevelopmental Outcomes in Patients with Hypoplastic Left Heart Syndrome
}

\author{
Elizabeth Hagan, PA-C \\ Harvey Feldman, MD
}

Nova Southeastern University

United States

CITATION: Hagan, E., Feldman, H. Neurodevelopmental Outcomes in Patients with Hypoplastic Left Heart Syndrome. The Internet Journal of Allied Health Sciences and Practice. April 2010. Volume 8 Number 2.

\begin{abstract}
Purpose: Hypoplastic Left Heart Syndrome (HLHS) accounts for $7-9 \%$ of all congenital heart diseases. It is fatal unless surgically corrected. Survival has improved overtime. However, neurodevelopmental outcomes have not been known until recently. This paper discusses the neurodevelopmental outcomes of patients with HLHS and the factors that influence these outcomes. Methods: A search of the literature was performed using several databases, medical textbooks and other on-line sources to locate research related to HLHS and neurodevelopment in HLHS. Results: Mean performance IQ scores tended to be lower than mean verbal IQ scores. Mean motor scores were below normal population values indicating impairment. Growth was impaired in many of those with HLHS. Factors that had negative impact on outcomes included deep hypothermic circulatory arrest (DHCA), cardiopulmonary bypass (CPB), longer hospital length-of-stay, seizures, and lower socioeconomic status (SES). Conclusions: The studies show that children with HLHS do have neurodevelopmental deficits. Intelligence, motor development and growth are all affected. Focus during care of these patients should be spent on improving the factors discussed that have been shown to have negative impacts on outcomes.
\end{abstract}

\section{INTRODUCTION}

Hypoplastic Left Heart Syndrome (HLHS) is a congenital condition that involves a spectrum of cardiac malformations characterized by underdevelopment of the left heart structures.1,2,3 HLHS is a lethal cardiac abnormality if not surgically corrected. ${ }^{3}$ Over time, survival rates in these patients have improved. However, neurodevelopmental outcomes in these patients have not been known until recently. This paper will describe the complex syndrome of HLHS and the surgical management of it and will focus on the neurodevelopmental outcomes of these patients. The specific outcomes used to measure neurodevelopmental status and the factors that influence these outcomes will be discussed.

\section{METHODS}

A thorough search of English language literature was conducted using Medline, EMBASE, ERIC, and Psyclnfo databases on all research related to HLHS. All of the research articles that looked at neurodevelopment in patients with HLHS were included. Subjects of all ages were included. No time frame for the search was pre-specified. However, the papers identified and reviewed ranged from 1995 through 2008. All surgical techniques used on these patients for the treatment of HLHS were included. A thorough search was also conducted in Cochrane and other databases for systematic reviews and meta-analyses on this topic. No such papers were found that provided neurodevelopmental data specifically on HLHS patients. Medical textbooks and online, continually-updated, reliable medical resources were also used for supportive data. Keywords used in the search included: Hypoplastic left heart syndrome, congenital heart disease, congenital heart surgery, neurodevelopment, development, neurocognitive, outcomes in hypoplastic left heart syndrome, cognitive development, Norwood, Fontan, and hemi-Fontan. 


\section{HYPOPLASTIC LEFT HEART SYNDROME}

In HLHS, there is marked hypoplasia of the left ventricle and ascending aorta. The aortic and mitral valves may be stenotic, atretic, or hypoplastic ${ }^{1,2,3}$ (Figure 1). The right ventricle becomes hypertrophied. The right atrium, right ventricle, pulmonary valve, and main pulmonary artery are dilated. ${ }^{1}$ This is because the right ventricle must function in place of the left ventricle by perfusing the lungs, via the branch pulmonary arteries, and the body, via the ductus arteriosus. If the pulmonary veins drain into the left atrium, an atrial septal defect must exist to allow pulmonary flow into the right atrium because blood cannot flow into the left ventricle..$^{1,2,3}$

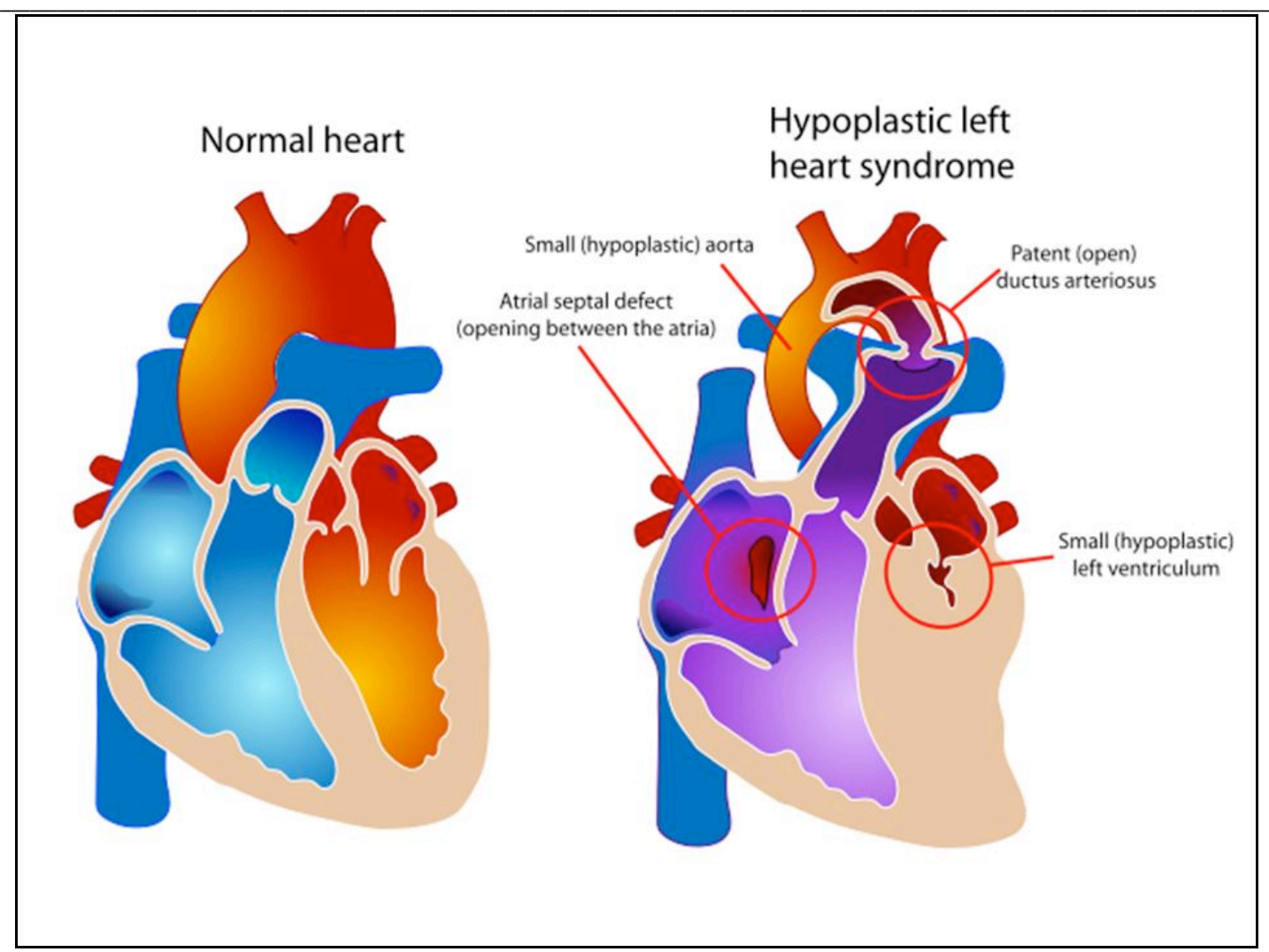

Figure 1. Illustration of a normal heart and a heart with HLHS.

In HLHS, blood returning from the lungs cannot pass from the left atrium to the left ventricle. It must pass through a defect in the atria septum to the right atrium. Blood is then pumped from the right atrium, to right ventricle, through the pulmonary artery and reaches the aorta through a patent ductus arteriosus so blood can be pumped to the rest of the body. The right ventricle is therefore responsible for pumping blood to the lungs and the body. ${ }^{21}$

After birth, pulmonary vascular resistance gradually decreases allowing a higher percentage of the right ventricular output to go to the lungs at the expense of systemic flow. Poor systemic perfusion can result in decreased coronary blood flow and cardiac output, metabolic acidosis, and end-organ damage. Alternatively, increased pulmonary vascular resistance may continue to elevate leading to decreased pulmonary blood flow causing profound hypoxemia. If there is spontaneous closure of the ductus arteriosus, the result is fatal. $1,2,3$

The incidence of HLHS is 0.16 to 0.36 per 1000 live births. ${ }^{2,3,4}$ It accounts for $7-9 \%$ of all congenital heart disease. It is the second most common congenital heart lesion presenting in the first week of life. ${ }^{3}$

Without surgical intervention, HLHS is fatal within the first few weeks of life.1,2,3,4 There are two treatment options for HLHS: cardiac transplantation or staged surgical reconstruction. Both are palliative, not curative. Cardiac transplantation was first successful in the early 1980s. It involves a donor heart and significant reconstruction of the recipient aortic arch using donor aorta. ${ }^{1}$ HLHS is one of the most common indications for infant heart transplantation. The drawback of transplantation is the shortage of donors. Approximately $20 \%$ of infants die while waiting for a donor. ${ }^{3}$ 
The other treatment option is a palliative three-stage repair. The first successful staged reconstructive repair for HLHS was completed and reported by Norwood and colleagues in 1983. ${ }^{5}$ The goal of surgical reconstruction is to eventually separate the pulmonary and systemic circulations. The right ventricle remains the systemic ventricle while blood flows to the lungs passively. ${ }^{3}$ The first stage, the Norwood procedure, is usually performed in the first few weeks of life. (Figure 2). The technical goals are aortic arch reconstruction to eliminate coarctation, align the single right ventricle with the systemic circulation, provide nonrestrictive coronary blood flow between the two atria by way of atrial septectomy, and optimize the pulmonary blood flow through an aortopulmonary shunt. Mortality rates are highest after this first stage due to the high systemic-to-pulmonary blood flow ratio with a high work load on the right ventricle and inefficient oxygen uptake by the lungs.3,4 The second stage, the hemi-Fontan or the bidirectional Glenn shunt, is performed between six and nine months of age.1,3 (Figure 3). This involves takedown of the previously placed shunt and creating anastomostomies between superior vena cava and the right pulmonary artery allowing venous blood to flow from the superior vena cava directly into pulmonary artery and then lungs. This stage is more physiologically stable than the first. 1,3,4 The third and final stage is the Fontan procedure performed about one year later. (Figure 4). Inferior vena cava blood is channeled into the pulmonary artery by creation of a tunnel in the right atrium. This results in complete separation of systemic circulation from the pulmonic circulation. ${ }^{1,3}$

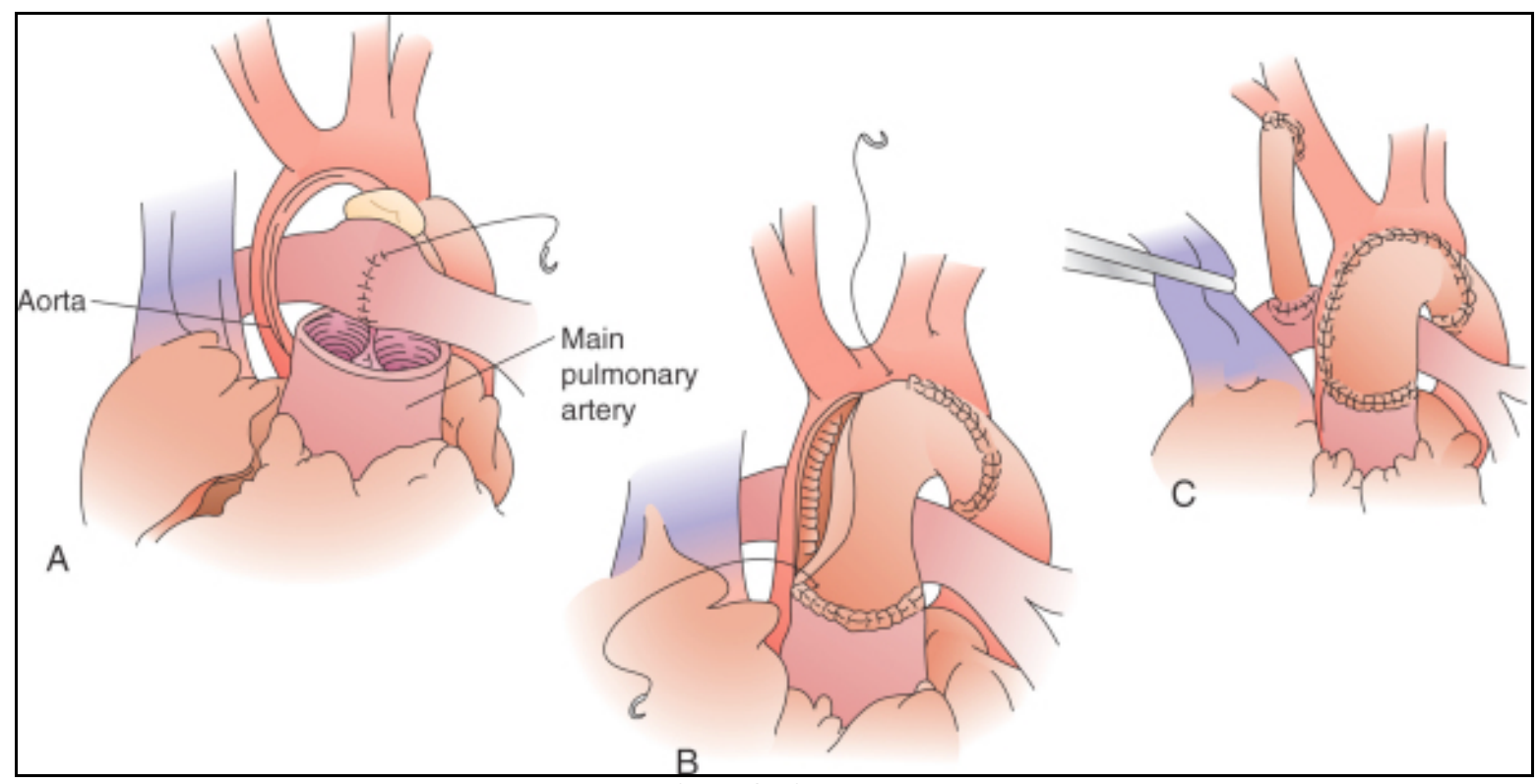

Figure 2. Illustration of HLHS after the Norwood Procedure.

In this procedure, the aorta is reconstructed, an atrial septectomy is performed so blood can flow freely between the atria, the ductus arteriosus is ligated, anastomostomies are created between the pulmonary artery and aorta to provide systemic blood flow and an aorta-to-pulmonary shunt is placed to provide pulmonic blood flow. 22 


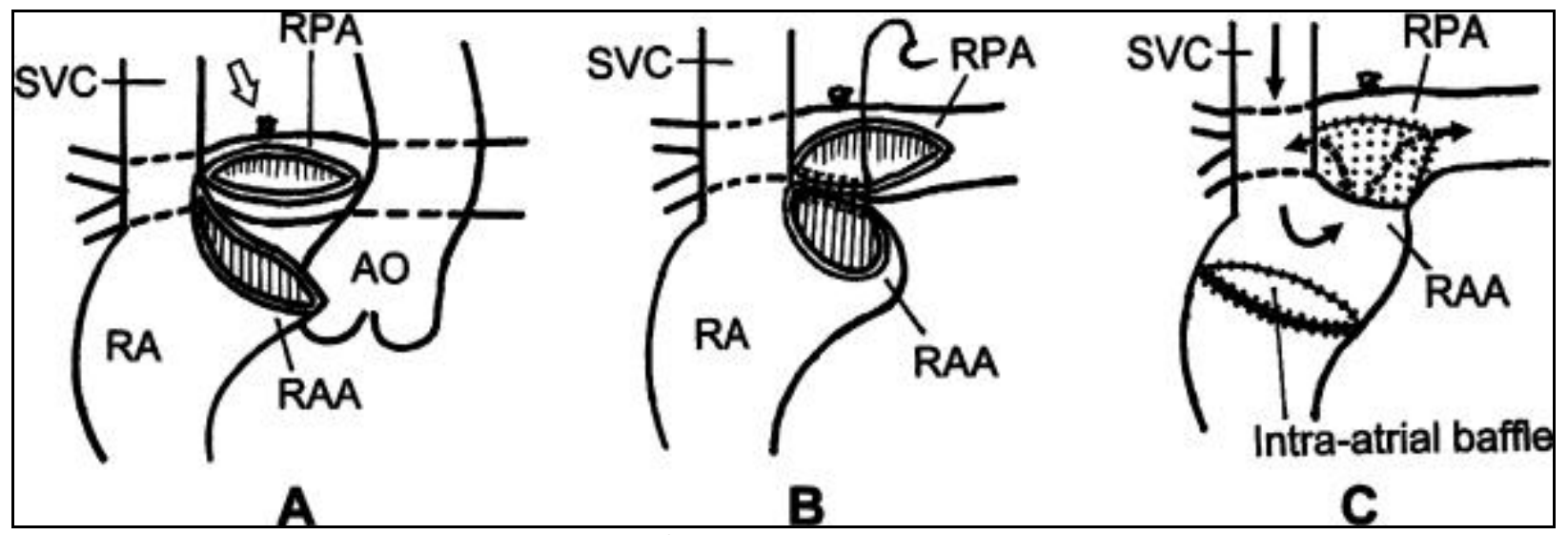

Figure 3.Illustration of the completed hemi-Fontan procedure.

Anastomostomies are created between the superior vena cava and right pulmonary artery so that venous blood return from the upper body can flow directly into the lungs. A patch is placed at the junction of the superior vena cava and right atrium. The previously placed aorta-to-pulmonary shunt is removed. ${ }^{23}$

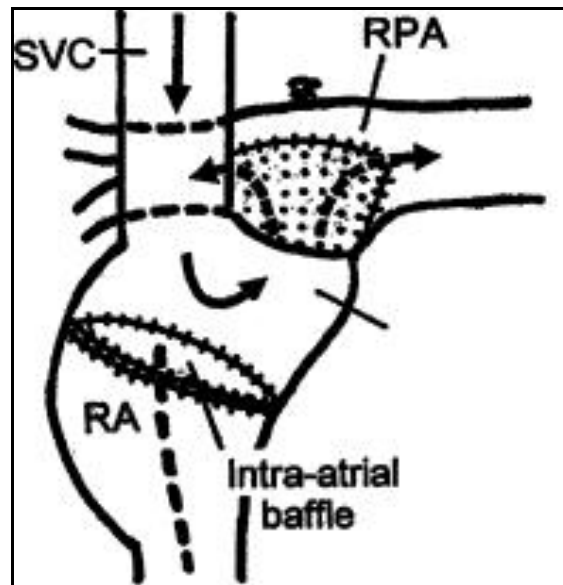

A

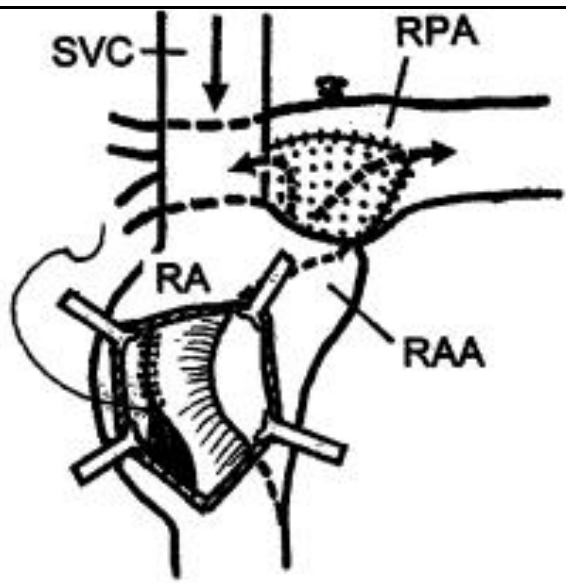

B

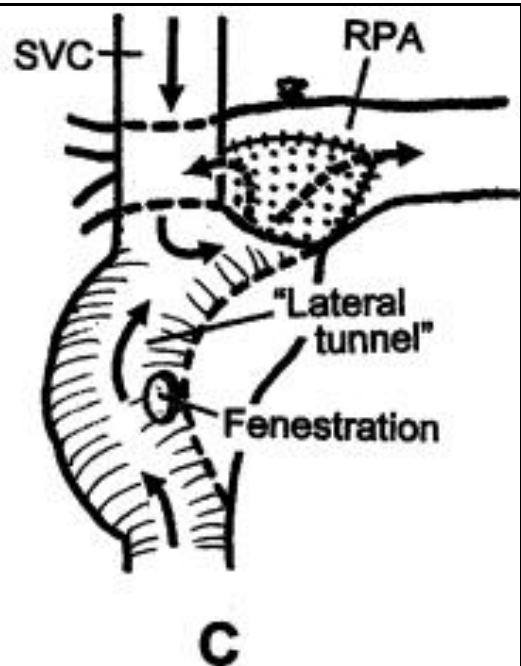

Figure 4. Illustration of the completed Fontan procedure.

In this procedure, a tube is placed in the right atrium that directs blood flow from inferior vena cava to the pulmonary artery. Systemic venous blood therefore flows passively to the lungs without going thorough a ventricle. ${ }^{23}$

Reported survival rates for cardiac transplantation and staged reconstruction vary but have improved over time. For those patients who survive the waiting period for cardiac transplantation, the survival rate at five years is nearly $80 \%$. Overall survival to the time of hospital discharge after the Norwood procedure is nearly $75 \%$. 3,6 Survival rates after the Hemi-Fontan/Glenn shunt and Fontan are $90-95 \%$. Survival rates after the completed three-staged reconstruction is $70 \%$ at five years. ${ }^{3}$

Although survival rates have improved overtime, little has been known regarding the cognitive, functional and neurological outcomes of these survivors until recently. Concerns have arisen in recent years regarding the neurodevelopmental outcomes in patients that have undergone surgical intervention for HLHS. Several studies have looked at these patients and found deficits in their development.

\section{NEURODEVELOPMENTAL OUTCOMES}

One of the main neurodevelopmental outcomes assessed in most of the studies is intelligence. Intelligence is assessed differently in infants as compared to school-aged children. For children aged three and older, all the studies to be discussed used 
variations of the Wechsler Intelligence test unless indicated otherwise. For those under three years of age (36 months), the Bayley Scales of Infant Development was used. This test is divided into two parts: the PDI (Psychomotor Development Index) which assesses gross and fine motor skills and the MDI (Mental Development Index) which assesses memory, problem-solving, number concepts, vocalization, and language skills.

The Wechsler Intelligence Test is comprised of two sections of subtests: verbal and performance. These two sections combined make up the full-scale IQ score. Scores are therefore given as performance IQ, verbal IQ, and full-scale IQ. The normal population value is $100+/-15$. Any score less than this is below normal population values. A score of 70 is two standard deviations below the normal value. Any score less than 70 indicates the child is functioning within the extremely low classification of intelligence or mental retardation. ${ }^{7}$

Overall, intelligence results have ranged from average to low average to varying degrees of mental retardation. ${ }^{8-15}$ Rogers et al were the first to report on the neurodevelopmental outcomes of infants (ages 11 to 67 months) with HLHS that had undergone surgical repair. ${ }^{8}$ Intelligence scores were calculated using combined McCarthy General Cognitive Index and the Bayley MDI scores. They found that seven of the eleven subjects had some degree of mental retardation - four moderate and three severe to profound.

Ikle et al divided subjects $(n=26)$ into two age groups to administer appropriate developmental testing for their ages. ${ }^{9}$ The results of the Bayley MDI for those less than 36 months old $(n=13)$ showed that scores were significantly lower in MDI than in the normal population. Two had scores less than 52 indicating severe developmental delay. They found that for those 36 months to six years of age $(n=13)$, the means (average scores) for verbal, performance and full-scale IQs were average to low average but significantly lower when compared with the normal population. Three children were borderline mentally retarded with IQ scores of 76,76 , and 71.

Of the 34 subjects, ages six to 13.6 years studied by Mahle et al in 2000, 10 children $(35.7 \%)$ scored in the borderline low range for full-scale IQ and five subjects (17.8\%) had mental retardation (scores less than 70). ${ }^{10}$ Median performance IQ scores were lower than the verbal IQ scores (83 vs. 90, respectively). In another study published in 2006 by Mahle et al, 48 subjects, ages eight to 17 years were tested and found to have intelligence scores significantly below population normative values ( $p<.01) .{ }^{11}$ The mean (average) full-scale IQ was 86 (range 57-115) indicating there were subjects with mental retardation (scores < 70) although the exact number is not reported.

Kern et al studied 14 subjects, ages 3.2-6.0 years, and found the mean full-scale IQ score to be 88 , which is below that of the normal population. ${ }^{12}$ Two patients had mental retardation (scores less than 70 ). Median performance IQ was lower than verbal IQ (83 vs. 91). Goldberg et al studied a total of 51 patients who had undergone the Fontan operation, those with HLHS and those without HLHS who had other forms of functional single ventricle malformations (which do not undergo the Norwood procedures). ${ }^{13}$ The HLHS group $(n=26)$ had mean IQ scores within the normal range and was not significantly different from those of the standard population. However, they scored significantly lower than the non-HLHS group on verbal, performance and full-scale IQ scores ( $p=.04, p=.02$ and $p=.01$ respectively). Also, three subjects had full-scale IQ scores less than 70 indicating significant impairment. Verbal scores were significantly higher than the performance scores $(p<.0001)$.

Creighton et al studied 62 survivors of infant cardiac surgery at ages two and five, 14 of which had undergone the Norwood procedure for HLHS. ${ }^{14}$ The Bayley test was used at age two and the Wechsler Intelligence test was used at age five. Those who had undergone the Norwood procedure had lower mean scores on the MDI portion of the Bayley test compared to three other groups who had undergone repair for other cardiac abnormalities (82 vs. 85 to 94) excluding those with chromosomal abnormalities. At age five, mean full-scale IQ was also lower in those who had undergone the Norwood procedure as compared to the others (85 vs. 91 to 101 ), excluding those with chromosomal abnormalities. Performance IQ was lower than normal population values and lower than verbal IQ (80 vs. 90) in those with HLHS.

Uzark et $\mathrm{al}^{15}$ and Wernovsky et $\mathrm{al}^{16}$ studied survivors of the Fontan operation and found that those with HLHS ( $\mathrm{H}=4$ and $n=5$, respectively) had lower mean IQ scores (88.4 and 70.4, respectively) than the normal population and compared to the rest of the subjects. The largest study that looked at neurodevelopmental outcomes in those with HLHS was by Tabbutt et al. ${ }^{17}$ There were 88 participants, ages $0.95-1.08$ years, and the Bayley Test was used to assess mental development. The median MDI score was 90 (range $50-129$ ) and $11 \%$ of the patients had mental retardation (scores $<70$ ).

All of these studies have shown that those with HLHS score no greater than average on intelligence testing per mean values. Also, all of these studies, except for that of Creighton et al and Uzark et al, included subjects with some degree of mental 
retardation. Creighton et al found a total of seven subjects with MDI scores less than 70 and three subjects with full-scale IQ scores less than 70 though did not indicate if these were patients with HLHS or subjects with other cardiac malformations. ${ }^{14}$ Uzark et al studied one subject with a mean IQ below the normal range, but did not indicate whether the patient had HLHS. ${ }^{15}$ Also there were only four subjects with HLHS in this study.

It is interesting to note that of the eight studies that used the Wechsler IQ tests, six studies found mean performance IQ scores to be lower than mean verbal IQ scores.9,10,11,12,13,14 However, Goldberg et al is the only study that indicates statistical significance $(p<.0001)$ when comparing verbal and performance IQ. The other two studies do not address this difference. 15,16 Rogers et al did not use the Wechsler IQ test. ${ }^{8}$

The findings from these studies show that results of the MDI scores using the Bayley Test are similar compared to the intelligence scores using the Wechsler tests. The consistency between these tests found throughout the studies makes the results more convincing.

All of the studies discussed had relatively small numbers of participants. However, all studies have shown similar consistent findings, namely that those patients with HLHS have mean IQ scores indicating no greater than average intelligence and that these patients are at risk for having mental retardation.

Motor skills is another neurodevelopmental outcome assessed in these patients. It is assessed by the Psychomotor Development Index (PDI) portion of the Bayley Test, the Beery-Buktenica Developmental Test of Visual Motor Integration (VMI) as well as a portion of the Vineland Adaptive Behavior Test. Results from the Bayley test will be discussed first. Rogers et al found gross motor delays in five of the eleven subjects. ${ }^{8}$ In the study by Creighton et al, when studied at age two, those who had undergone the Norwood procedure (those with HLHS) had lower mean PDI scores compared to the other subjects (69 vs. 82 to 101) excluding those with chromosomal abnormalities. ${ }^{14}$ The mean PDI score in those with HLHS indicates there was motor impairment in some of the infants. Results from Tabbutt et al showed that the PDI scores ranged from 50-117 with a median score of 73.16 Forty-two (48\%) had scores less than 70 indicating significant motor impairment. Ikle et al also found PDI scores significantly lower overall when compared with a normal population. ${ }^{8}$ Two children had PDI scores (as well as MDI scores) less than 52 indicating severe developmental delay. These four studies all had the same results which showed that motor skills were overall below normal population values.

Visual Motor Integration (VMI) assesses visual-spatial and visual-motor integration ability. It is designed to identify problems with visual perception, motor coordination, and visual-motor integration such as hand-eye coordination. The mean value is $100+/-15$ and anything less indicates motor impairment.

Results of VMI testing by Mahle et al (2000) showed that the median score was 85.5, range 57-109, indicating many of the subjects had lower than normal values and some (an exact number is not given) had significant impairment in motor capabilities. ${ }^{10}$ In Mahle et al (2006), the mean VMI scores were significantly below normal population values $(p<.01) .{ }^{11}$ Mean VMI scores were $75+/-16$ for those who had undergone the Norwood procedure, $77+/-12$ for those who had undergone cardiac transplantation and $76+/-14$ for both combined. In Kern et al, the mean VMI score was $82+/-16$ which is also below normal population values. ${ }^{12}$ The study by Creighton et al showed that mean VMI scores for those who had undergone the Norwood procedure were also below normal population values and lower than the other subjects ( 80 vs. 83 to 103 ), excluding those with chromosomal abnormalities. ${ }^{14}$ Uzark et al also found a mild weakness in VMI with mean score of 94.8. ${ }^{15}$ However, this includes all patients in the study, those with HLHS and those without (who have undergone Fontan procedure for other single ventricle malformations).

The Vineland Adaptive Behavior Scales (VABS) provides information regarding adaptive behavior. Information is obtained through parent interviews. The categories are skills of daily living, communication, social interactions and motor capabilities. Individual scores are obtained from each and an overall score is compiled. The standard score is $100+/-15$.

In Kern et al, the median adaptive behavior score was $91+/-20$ with social skills the strongest (median score of 103) and motor skills the weakest (median score of 85 ). ${ }^{12}$ When the VABS scores were compared with the control group in this study, there was a statistically significant difference in adaptive behavior $(p=.025)$. All scores were statistically lower for those with HLHS, with motor skills showing the greatest discrepancy. Goldberg et al found that the mean VABS scores were within normal ranges for both groups, those with HLHS and those without HLHS (who have other forms of functional single ventricle malformations). ${ }^{13}$ The only exception was motor skills which were below the normal range. Communication scores were significantly higher than motor scores $(p<.002)$. HLHS patients tended to have lower VABS scores than the non-HLHS group. Ikle et al used a 
downward extension of the VABS called the Vineland Social-Emotional Early Childhood Scale which excludes motor skills. ${ }^{9}$ This is probably because they used the Bayley test to assess motor skills. Results showed that the means for the Vineland were significantly lower than expected for communication and daily living skills and for the overall adaptive behavior score, but not for socialization. It is important to remember in interpreting all of the results of the VABS that the scores rely heavily on reports from the parents.

Although there were three different tests used to assess motor function in these studies, all of the results were similar. These studies showed that motor function scores were below normal population values indicating impairment in motor capabilities.

Motor development has been shown to be more affected than intelligence. Tabbutt et al found the PDI scores to be much lower than the MDI scores of the Bayley test indicating motor development to be more negatively affected. ${ }^{17}$ Creighton et al also showed mean PDI scores to be lower than mean MDI scores in those who had undergone the Norwood procedure. Kern et al12 and Goldberg et al ${ }^{13}$ also found motor skills to be most impaired in the VABS test when assessing adaptive behavior. It is possible that the reasons for greater motor impairment are related to the limitation of activity due to impaired cardiac function. Also, the cumulative recovery time from the surgeries may be great enough to have significant impairment on normal motor development.

Growth is another neurodevelopmental outcome assessed in these HLHS patients. Rogers et al found that upon follow-up when neurodevelopment was assessed, five of the eleven children were underweight with one having a weight-to-height ratio less than the fifth percentile. ${ }^{8}$ Eight children had acquired microcephaly. In Ikle et al, there was a high rate of growth failure. ${ }^{9}$ Median weight was at the seventh percentile (range $<5 \%$ to $55 \%$ ) and median height was at the 12.5 percentile (range $<5 \%$ to $65 \%$ ). Of the 26 total subjects, 11 had weights less than the fifth percentile. Four of these subjects were aged two to three years and seven of these subjects were aged five to six years. Growth was poor in the younger subjects and the older subjects indicating that growth may remain delayed. In the study by Creighton et al, five of the 14 patients with HLHS had heights or weights less than the fifth percentile at age five..$^{14}$ Kelleher et al studied growth outcomes at length. ${ }^{18}$ There were 50 infants who had undergone stage one and stage two surgical repairs for HLHS from January 1995 to June 1999. Information was gathered from medical charts to assess growth and nutritional status from initial hospitalization and discharge and admission for the second surgery. Nutritional status on the first admission was within normal limits for all subjects. Median discharge weight was unchanged from admission weight at $3.4 \mathrm{~kg}$. At admission for the second surgery, $50 \%$ of all infants were severely underweight.

These four studies found growth to be impaired in many of the patients with HLHS. Although some children had average growth percentiles at the time of evaluation, many of them were underweight. Also, growth may continue to lag behind normal values as the children grow older.

As previously mentioned with the discussion of intelligence, all of these studies that discussed motor development and growth had relatively small numbers of participants ranging from four to 88 participants. It would be interesting to see if the results of the studies were still statistically significant with a greater sample size. The only study that used a control group was Mahle et al (2000) and a power analysis was not performed to determine if the sample size was sufficient to rule out a false positive result. However, all of these studies had similar findings in motor development and growth.

\section{FACTORS THAT IMPACT NEURODEVELOPMENTAL OUTCOMES}

Factors that affected neurodevelopmental outcomes varied between the studies. However, there were some commonalities among them. Surgical repair for HLHS involves deep hypothermic circulatory arrest (DHCA) and cardiopulmonary bypass (CPB). CPB exposes infants to harmful effects that are more pronounced than those in adults because of the immaturity of peripheral tissues and organ function. ${ }^{19}$ CPB has been associated with hypoglycemia, increased stress response on the body, hematologic and renal effects and negative effects on lung and cardiac tissue. ${ }^{19}$ CPB in combination with DHCA has been shown to affect the CNS as well. ${ }^{19}$ During DHCA, there is absolutely no blood flow occurring to the brain or body. Evidence of neurologic injury is observed in as many as $25 \%$ of infants who undergo DHCA. ${ }^{19}$

CPB and DHCA are the main surgical factors that have been evaluated for their possible influence on neurodevelopmental outcomes. This is because they have been associated with neurodevelopmental deficits in children who have undergone other types of cardiac surgery. Mahle et al (2000) found that longer CPB duration was associated with lower performance IQ scores ( $p$ $=.007) .{ }^{10}$ However, neither duration of DHCA at the time of stage one repair nor cumulative DHCA time for all operations was predictive of full-scale IQ scores. Kern et al, ${ }^{12}$ Goldberg et al, ${ }^{13}$ Uzark et al15 and Wernovsky et al ${ }^{16}$ all found opposite results. They found that CPB time did not correlate with intelligence, but DHCA did correlate negatively with intelligence. Kern et al found that DHCA correlated negatively with full-scale IQ scores $(p=.014)$ due to the strong negative correlation with performance IQ $(p$ 
$=.004)$. Duration of DHCA did not correlate with verbal IQ $(p=.12)$. Goldberg et al also found that the use of DHCA did correlate negatively with intelligence $(p=.002)$. Uzark et al found that those who had undergone Norwood surgery prior to the Fontan (all those with HLHS) which used DHCA had lower mean IQ scores $(88.4 ; p=.06) .15$ In the study by Wernovsky et al, DHCA in patients with HLHS, who all underwent surgery that involved DHCA prior to the Fontan, was significantly associated with lower full-scale IQ $(p=.002) .{ }^{16}$ Other studies, including Mahle et al (2006), Ikle et al, Creighton et al. and Tabbutt et al found no relation of developmental test scores with use of CPB or DHCA.9,11,14.17

The length of time a patient is under DHCA would logically seem to have an impact on neurodevelopmental outcomes. In Mahle et al (2000) where DHCA was not associated with neurodevelopmental outcomes, the average duration was one hour and there was little variation among patients in the amount of time under DHCA. ${ }^{10}$ In Mahle et al (2006), which found no relation of DHCA to outcome, duration of DHCA was similar. ${ }^{11}$ Mean DHCA times were 59 minutes for those that underwent Norwood procedure and 52 minutes for those that underwent transplantation. The median duration of DHCA in Ikle et al and Tabbutt et al was 64.7 +/-17.4 minutes and 65 minutes (range 5-127), respectively.9.17 These time periods under DHCA were not associated with neurodevelopmental outcomes yet longer in time than the DHCA duration in Kern et al and Uzark et al where DHCA duration was associated with outcomes. The average time of DHCA in the patients studied by Kern et al and Uzark et al, where DHCA was associated with outcomes, was 56 minutes and $47.8+/-8.2$ minutes, respectively. ${ }^{12,15}$ In Goldberg et al, DHCA times for those with HLHS were not given; however duration of DHCA was not associated with intelligence scores. ${ }^{13}$ Only use of DHCA was associated with intelligence scores. Creighton et al and Wernovsky et al did not make note of DHCA duration. ${ }^{14,16}$ These finding show that the duration of DHCA was not the reason for studies finding a positive correlation of DHCA with neurodevelopmental outcomes nor the reason for a lack of a correlation of DHCA to outcomes.

Another factor that has affected neurodevelopmental outcomes is hospital stay. Tabbutt et al found that a longer post-op lengthof-stay for the stage one repair was associated with abnormal or suspect neuromuscular exam results $(p=.05) .{ }^{17}$ Mahle et al (2006) also found that prolonged hospital stay (> 40 days) was associated with lower median full-scale IQ scores when compared with those who stayed less than 40 days (78 vs. 92, respectively; $p=.003$ ). ${ }^{11}$ Longer length-of-stays were also associated with lower verbal IQ $(p=.01)$ and lower performance IQ scores $(p=.01)$. Kelleher et al found hat longer length-ofstay and longer ICU stay had a negative impact on growth. ${ }^{18}$ Weight-for-age Z-scores were lower in those with longer length-ofstays in the hospital and longer ICU stay. Ikle et al found that length of time waiting for transplantation correlated negatively with neurodevelopmental outcome. ${ }^{9}$ When the IQ or MDI of the eight infants who waited four months or greater for transplantation as compared to 18 others who were given transplantation sooner, the difference was statistically significant $(p<.004)$.

Seizures are another factor that have been associated with neurodevelopmental outcomes. Mahle et al (2000) found that preoperative seizures were associated with lower full-scale IQ scores $(p=.01)$, lower verbal IQ scores $(p=.004)$ and lower performance IQ scores $(p=.02)$. ${ }^{10}$ Goldberg et al found that perioperative seizures were associated with lower neurodevelopmental scores.13 The overall intelligence score for those who had seizures, which only occurred in those with HLHS, was 82.4+/-17.4 as compared to those who had not had seizures whose score was $102.2+/-6.1(p<.03)$.

Socioeconomic status (SES) is another factor that has been evaluated in relation to neurodevelopmental outcomes. Wernovsky et al found that higher SES was a strong predictor of higher IQ $(p<.001) .{ }^{16}$ However, this correlation includes all subjects in the study, those with HLHS and those without. Ikle et al and Mahle et al (2000) found that SES did not affect neurodevelopmental outcomes. .10

There are other factors that have been found to affect outcomes. In terms of growth, Ikle et al found that some of those with growth failure had feeding problems such as reflux and the need for tube feeds and some required interval rehospitalization. ${ }^{9}$ Kelleher et al found that in addition to length-of-stay and ICU stay, other factors that adversely affected growth, before adjusting for weight-for-age, were longer duration of mechanical ventilation, higher calorie-per-ounce feedings and more days in the hospital before stage one surgery. These factors were associated with lower weight-for-age $Z$ scores at discharge. ${ }^{18}$ The strongest correlation of discharge nutritional status was admission nutritional status. After adjusting for weight-for-age at admission, those with longer length of stay, longer length of ICU stay, shorter duration of parenteral nutrition and higher diuretic dosage at discharge had lower weight-for-age $Z$ scores at discharge. At admission for the second surgery, lower weight-for-age $Z$ scores were associated with more frequent readmissions, higher oxygen saturation at discharge after the first surgery and shorter duration of parenteral nutrition in addition to longer length-of-stay for the first surgery. After adjusting for weight-for-age at the time of discharge for the first surgery, those patients with fewer calories per ounce of their enteral nutrition formula at discharge, more right ventricular dysfunction before the second surgery, more frequent admissions, and higher oxygen saturation at discharge had lower weight-for-age Z-scores at the second surgery. 
Impairment in growth may be due to several factors that have not been discussed. There is an increased metabolic rate in children with HLHS and other congenital heart diseases due to the increased energy expenditure associated with tachycardia or tachypnea. There may also be inadequate nutrient intake related to fatigue, dyspnea, reflux or loss of appetite. One or a combination of these can lead to protein-energy malnutrition and growth impairment. ${ }^{20}$

Other factors that have negatively affected neurodevelopmental outcomes include preoperative intubation and genetic syndromes. ${ }^{17}$ Surgical strategy (transplantation versus Norwood procedure) was not associated with any developmental test outcome in the study by Mahle et al (2000). ${ }^{10}$

\section{DISCUSSION}

Overall, survival has improved in patients with HLHS who have undergone surgery. Before surgical treatment was available, HLHS was responsible for $25 \%$ of cardiac deaths in the neonatal period. ${ }^{3}$ Throughout the years, changes have been made in the care and management of patients preoperatively, intra-operatively, and post-operatively. Also, there has been increased knowledge and awareness of HLHS and the increased frequency of prenatal diagnosis. There have been enhancements in the skills of the surgeons and advancements in the procedures performed due to what has been learned over the years.

Although survival has much improved in patients with HLHS, neurodevelopmental outcomes appear to have only slightly improved. When looking at the first study that assessed neurodevelopment in these patients (Rogers et al, 1995), there was a high prevalence $(64 \%)$ of developmental disabilities. ${ }^{8}$ The most recent study by Tabbutt et al divided patients between two time eras, April 1998 through January 2001 and January 2001 through March 2003.17 Results showed that the patients in the later era had better MDI $(p=.079)$ and PDI $(p=.084)$ scores. Overall however, $11 \%$ had mental retardation per MDI and $48 \%$ had severe psychomotor developmental delay. Most of the other studies evaluated patients over a several year period from the late 1980 s to early 1990s. All studies had average scores that were below normal population values and two to three patients (8-18\%) with mental retardation.

The results of these studies show that neurodevelopmental deficits do still exist and most of these children have deficiencies in more than one area. Intelligence, motor development, and growth are all affected. There is no universal factor among the studies that has been shown to affect outcomes. There are multiple factors that come into play in the outcomes of these children. Whatever the reasons, these children do, on average, have impairments in their neurodevelopment.

\section{CONCLUSION}

With outcomes in survival improving, the focus now needs to be on improvements in neurodevelopmental outcomes. Reviewing the commonalities in factors that have been shown to affect outcomes in previous studies and trying to improve in these areas is the first step towards improving neurodevelopmental outcomes. Variables that occur not only intra-operatively with surgical techniques, but also preoperatively and postoperatively, need to be further assessed and improved. There are unrecognized factors that determine outcomes such as genetics and unknown genetic syndromes associated with HLHS. There are also factors such as the influence of family dynamics and quality of care of the patients by their families. These are factors that practitioners cannot control. The factors that we do have control over need to be more thoroughly evaluated for changes or improvements. Success cannot be defined by survival statistics alone. Success must encompass the patient as a whole.

\section{REFERENCES}

1. Crawford MH, DiMarco JP, Paulus WJ, et al. Cardiology $2^{\text {nd }}$ Edition. Philadelphia, PA: Elsevier Limited; 2004.

2. Jonas RA. Comprehensive Surgical Management of Congenital Heart Disease. London, England: Arnold; 2004.

3. Syamasundar RP. Hypoplastic Left Heart Syndrome. Emedicine. http://www.emedicine.com/ped/TOPIC1131.htm. Updated August 15, 2006. Accessed September 25, 2008.

4. Hennein HA, Bove EL, eds. Hypoplastic Left Heart Syndrome. Armonk, NY: Futura; 2002.

5. Norwood WI, Lang P, Hansen DD. Physiological repair of aortic atresia-hypoplastic left heart syndrome. N Engl J Med. 1983;308:23-26.

6. Zieve D, Fogal MA. Hypoplastic left heart syndrome. http://www.nlm.nih.gov/medlineplus/ency/article/001106.htm. Updated December 12, 2007. Accessed October 14, 2008.

7. Weiss LG, Prifitera A, Holdnack JA, et al. WISC-IV Advanced Clinical Interpretation. Elsevier Inc; 2006.

8. Rogers BT, Msall ME, Buck GM, et al. Neurodevelopmental outcome of infants with hypoplastic left heart syndrome. Journal of Pediatrics. 1995;126(3):496-498. 
9. Ikle L, Hale K, Fashaw L, et al. Developmental outcome of patients with hypoplastic left heart syndrome treated with heart transplantation. Journal of Pediatrics. 2003;142: 20-25.

10. Mahle WT, Clancy RR, Moss EM, et al. Neurodevelopmental outcome and lifestyle assessment in school-aged and adolescent children with hypoplastic left heart syndrome. Pediatrics. 2000;105(5):1082-1089.

11. Mahle WT, Visconti KJ, Freier MC, et al. Relationship of surgical approach to neurodevelopmental outcomes in hypoplastic left heart syndrome. Pediatrics. 2006; 177(1): e90-e97.

12. Kern JH, Hinton VJ, Nereo NE, et al. Early neurodevelopmental outcome after the Norwood procedure for hypoplastic left heart syndrome. Pediatrics. 1998;102(5):1148-1152.

13. Goldberg CS, Schwartz EM, Brunberg JA, et al. Neurodevelopmental outcome of patients after the Fontan operation: A comparison between children with hypoplastic left heart syndrome and other functional single ventricle lesions. Journal of Pediatrics. 2000; 137(5):646-652.

14. Creighton DE, Robertson CMT, Sauve RG, et al. Neurocognitive, Functional and Health Outcomes at 5 Years of Age for Children at 6 Weeks of Age or Younger. Pediatrics, 2007; 120(3):e478-e486.

15. Uzark K, Lincoln A, Lamberti JJ, et al. Neurodevelopmental outcomes in children with Fontan repair of functional single ventricle. Pediatrics. 1998;101(4):630-633.

16. Wernovsky G, Stiles KM, Gauvreau K, et al. Cognitive development after the Fontan operation. Circulation. 2000;102(8):883-889.

17. Tabbutt S, Nord AS, Jarvik GP, et al. Neurodevelopmental outcomes after staged palliation for hypoplastic left heart syndrome. Pediatrics. 2008;121(3):476-483.

18. Kelleher DK, Laussen $P$, Teixeira-Pinto A, et al. Growth correlates of nutritional status among infants with hypoplastic left heart syndrome after stage one Norwood procedure. Nutrition. 2006;22:237-244.

19. Alsoufi B. Hypothermia, Circulatory Arrest and Cardiopulmonary Bypass. http://www.emedicine.com/ped/TOPIC2813.HTM. Updated: August 1, 2006. Accessed: December 6, 2008.

20. Forchielli ML, McColl R, Walker WA, et al. Children with congenital heart disease: A nutrition challenge. Nutrition Reviews. 1994:52(10):348-353.

21. Ruiz M. http://commons.wikimedia.org/wiki/File:Hypoplastic left_heart_syndrome.svg

1. Updated July 16, 2007. Accessed January 3, 2009.

22. Townsend CM, R. Beauchamp DB, Evers M, Mattox K. Sabiston Textbook of Surgery, 18th ed (online). Saunders Elsevier; 2007: Figure 60-37.

23. Park MK. Pediatric Cardiology for Practitioners, 5th ed (online). Mosby Elsevier; 2008: Figures 14-39 \& 14-40. 